\title{
Minimum Dipole Moment Required to Bind an Electron-Molecular Theorists Rediscover Phenomenon Mentioned in Fermi-Teller Paper on Another Subject Twenty Years Earlier
}

\author{
J. E. Turner
}

\section{OAK RIDGE NATIONAL LABORATORY}




\section{DISCLAIMER}

This report was prepared as an account of work sponsored by an agency of the United States Government. Neither the United States Government nor any agency Thereof, nor any of their employees, makes any warranty, express or implied, or assumes any legal liability or responsibility for the accuracy, completeness, or usefulness of any information, apparatus, product, or process disclosed, or represents that its use would not infringe privately owned rights. Reference herein to any specific commercial product, process, or service by trade name, trademark, manufacturer, or otherwise does not necessarily constitute or imply its endorsement, recommendation, or favoring by the United States Government or any agency thereof. The views and opinions of authors expressed herein do not necessarily state or reflect those of the United States Government or any agency thereof. 


\section{DISCLAIMER}

Portions of this document may be illegible in electronic image products. Images are produced from the best available original document. 
Printed in the United States of America. Available from Natınnal I eschnical Intormation Sorvice

U.S. Department of Commerce

5285 Port Royal Road, Springfield, Virginia 22161

Price: Printed Copy $\$ 4.00$; Microfiche $\$ 2.25$

This report was prepared as an account of work sponsored by the United States Government. Neither the United States nor the Energy Research and Development Administration/United States Nuclear Regulatory Commission, nor any of their employees, nor any of their contractors, subcontractors, or their employees, makes any warranty, express or implied, or assumes any legal liability or responsibility for the accuracy, completeness or usefulness of any information, apparatus, product or process disclosed, or represents that its use would not infringe privately owned rights. 
Contract No. W-7405-eng-26

HEALTH PHYSICS DIVISION

MINIMUM DIPOLE MOMENT REQUIRED TO BIND AN ELECTRON-MOLECULAR THEORISTS REDISCOVER PHENOMENON MENTIONED IN FERMI-TELLER PAPER ON ANOTHER SUBJECT TWENTY YEARS EARLIER

J. E. Turner

JULY 1976

This report was prepared as an account of work

the United States United States Government. Neither

Research and Development Administration, nor any of dhes enployes, not any of theis contractors. Wubcontactors, or their employees, makes any

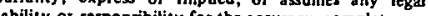

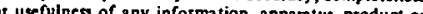
ofed, or represents, that its use would not infringe privetely owned rights. 


\begin{abstract}
MINIMUM DIPOLE MOMENT REQUIRED TO BIND AN ELECTRON-MOLECULAR THEORISTS REDISCOVER PHENOMENON MENTIONED IN FERMI-TELLER PAPER ON ANOTHER SUBJECT TWENTY YEARS EARLIER
\end{abstract}

\author{
J. E. Turner \\ Health Physics Division \\ Oak Ridge National Laboratory \\ Oak Ridge, TN 37830
}

\begin{abstract}
Work leading to the discovery of the minimum dipole moment for electron binding, $D_{\text {min }}=0.639 \mathrm{ea}_{0}$ (atomic units), by several groups in 1967-68 is described. It was subsequentiy learned that this number had been published in 1947 by Fermi and Teller, who did not, however, indicate how they derived it. The author has found a numerical solution in Fermi's notebooks from 1946-50 at the University of Chicago Library. Fermi's work is described and presented here with relevant material from his notebooks.
\end{abstract}


This article is not about one of the great discoveries in physics. Instead, it tells the history of a small discovery. It should be of some general interest because of the physics involved, and it will also serve to record a rather strange series of events that ended last fall in Fermi's Notebooks at the University of Chicago Library.

\section{ELECTRON CAPTURE BY ROTATIONAL EXCITATION OF POLAR MOLECULES}

When I reported for work in the Health Physics Division at the Oak Ridge National Laboratory in February 1962, my supervisor, G. S. Hurst, suggested several theoretical problems that I might work on. One experiment, which he and his colleagues had carried out, particularly intrigued me. Hurst's group had performed electron-swarm experiments and measured drift velocities in mixtures consisting of small amounts of a polar gas added to non-polar ethylene. They determined the ratio of thermal-energy electron scattering cross sections for various polar molecules relative to ethylene and compiled these data over a range of molecular dipole moments. A pint of experimental results ${ }^{\prime}$ is shown iil Figure 1. The straight line gives the theoretical cross section based on a first Born-approximation calculation by Altshuler of electron scattering from a stationary point dipole. ${ }^{2}$ Although general agreement between experiment and theory was good for most molecules, a discrepancy was found with $\mathrm{H}_{2} \mathrm{O}, \mathrm{D}_{2} \mathrm{O}$, and $\mathrm{H}_{2} \mathrm{~S}$, which exhibited cross sections about a factor of two greater than expected. Hurst suggested that the torque exerted on the molecular dipole by an electron might cause a rotational excitation of the molecule with temporary capture of the electron. The excited ion would subsequently release the electron back into the swarm. 
ORNL-LR-DWG-75023

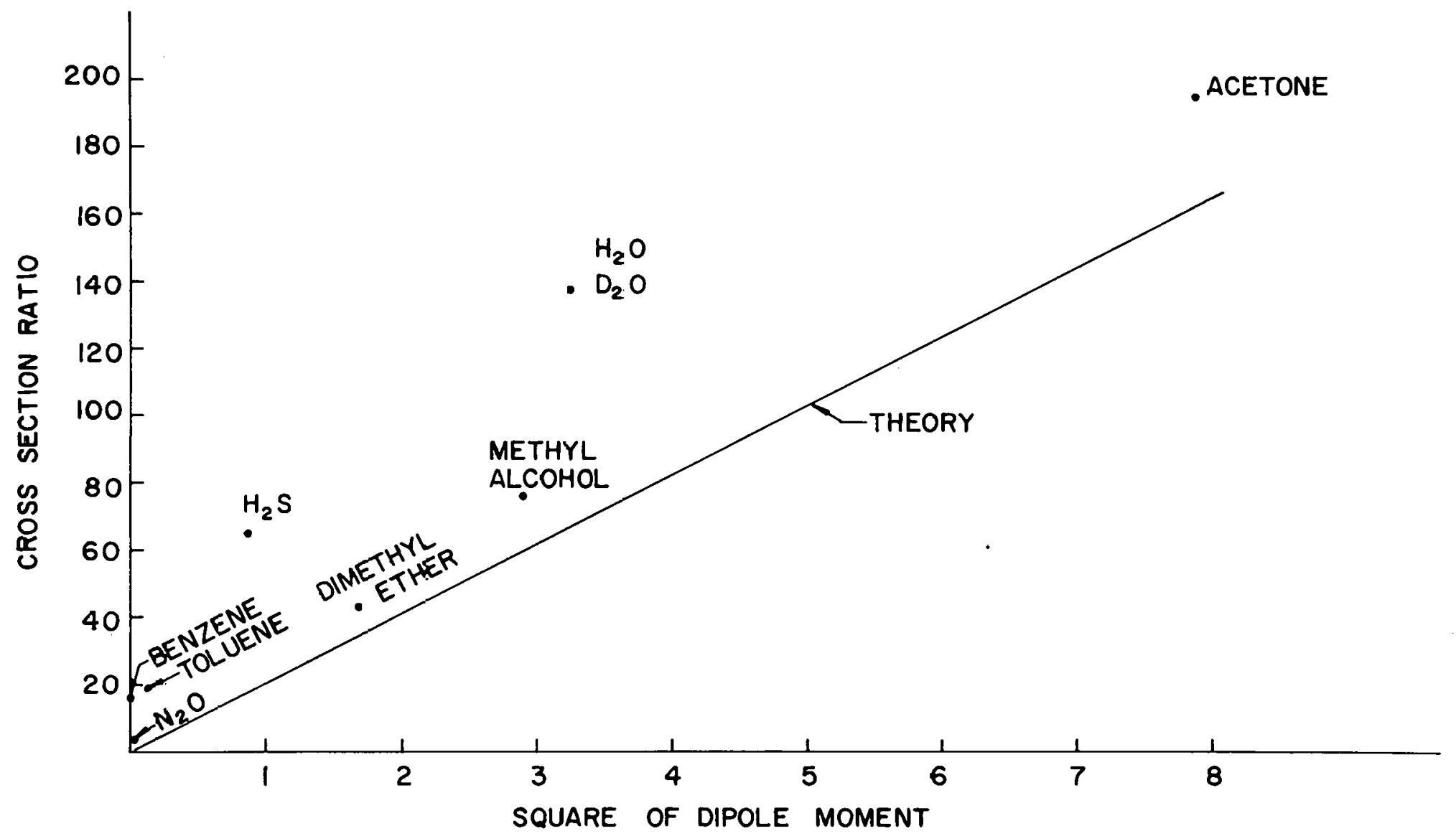

Fig. 1 
The process is illustrated for the water molecule by writing

$$
\mathrm{H}_{2} \mathrm{O}+\mathrm{e}^{-} \neq \mathrm{H}_{2} \mathrm{O}^{-*} \text {. }
$$

This quasi-trapping and subsequent release of the electron would, in effect, increase the amount of elastic scattering and might account for the observed increase in the cross section over that calculated by Altshuler.

The electron-dipole system is shown schematically in Fig. 2. The electron, with charge $-e$, moves in the field of two stationary charges, $\pm q$, separated by a distance $R$. The coordinates $r^{\prime}$ and $r^{\prime \prime}$ give the distances of the electron from the ends of the dipole. The dipole moment is given by $D=q R$. Detailed calculations ${ }^{3}$ indicated that unimolecular electron capture by rotational excitation could occur with a high probability, provided the molecule possesses a large permanent dipole moment and a small moment of inertia, I. The latter property is important for two reasons. First, the capture probability varies as $I^{-2}$. Second, the rotational energy-level spacings are of the order of thermal energies, $k T$, only if the moment of inertia is small. Since the selection rules for changes in the rotational angular momentum $\mathrm{J}$ of the molecule are $J= \pm 1$ and \pm 2 , rotational excitation will be accompanied by capture into a negative-energy electronic state only when $I$ is small. There are just a few molecules that possess this combination of large $\mathrm{D}$ and small I-- $\mathrm{NH}_{3}, \mathrm{HF}, \mathrm{HCl}, \mathrm{LiH}, \mathrm{LiF}$, and $\mathrm{H}_{2} \mathrm{O}_{2}$, in addition to $\mathrm{H}_{2} \mathrm{O}, \mathrm{D}_{2} \mathrm{O}$, and $\mathrm{H}_{2} \mathrm{~S}$.

The above theoretical results were worked out in a Born-Oppenheimer approximation, in which the motion of the electron is calculated for fixed values of the nuclear coordinates. In the course of the investigation, 
I became interested in the general problem of the bound states of an electron in the field of a stationary, permanent electric dipole. In calculating the capture probability I had assumed that the electron would be temporarily bound in a combination of $S$ and $P$ states with a hydrogenic ground-state radial dependence. Such a function gives a negative expectation value of the electron's energy for dipole moments $D>0.968 \mathrm{ea}_{0}, 4$ somewhat larger than the dipole moment of $\mathrm{H}_{2} \mathrm{O}, 0.728 \mathrm{ea}_{0}$. The question naturally arose whether a bound state exists for arbitrarily small values of the dipole moment.

\section{MINIMUM DIPOLE MOMENT}

Wallis, Herman, and Milne had calculated the ground-state and several excited-state energy eigenvalues over the range of the dipole moments from 0.840 to $30 \mathrm{ea}_{0^{\circ}}{ }^{5}$ They set the dipole charges equal to the electronic charge, $\pm e$, separated by a distance $R$, so that $D=e R$. When one goes to larger and larger values of the dipole momemt, the electron tends more and more to be associated with the positive charge of the dipole. At large $R$ the system is equivalent to a hydrogen atom perturbed by a distant, stationary electronic charge. The ground-state binding energy when $R=30 \mathrm{ea}_{0}$ is $0.933 \mathrm{Ry} .{ }^{6}$ In going to small values of $\mathrm{R}$, Wallis, Herman, and Milnes reported that the convergence of one of the continued fractions in their calculations became poor. The binding energy for $D=0.840$ ea $_{0}$, where their computations leave off, is $4.64 \mathrm{x}$ $10^{-5}$ Ry. As to whether there is a minimum value $R>0$ needed to bind the electron, they conclude, "One still cannot tell for sure whether $E_{B}$ [the binding energy] is greater than zero for every $R$ greater than zero..." 
In the summer of 1964, Kenneth Fox joined me in working on the electron-dipole system. We solved the problem by Hamilton-Jacobi theory and found that, classically, there is always a bound state, no matter how small the dipole moment. ${ }^{7,8}$ We also worked out a quantummechanical solution in the WKB approximation ${ }^{9}$ and found that a non-zero minimum value is required to give a bound state. The WKB solution gives bound states only when $D>0.860 \mathrm{ea}_{0}$. While we knew already from the Wallis, Herman, and Milne work that negative-energy states occur for this value of $D$, we felt that the requirement for a minimum dipole moment greater than zero must be due to the quantum-mechanical nature of the problem rather than the WKB method. Fox and I also carried out a variational calculation, assuming that the ground-state wave function is a sum of $S$ and $P$ states and has a radial dependence of the form $\exp \left(-\alpha r^{t}\right)$, where $\alpha$ and $t$ are adjustable parameters. When $\alpha$ and $t$ tend toward zero, this function represents a weakly bound state, spread out over a large region of space. In this limit we found 10 that a bound state exists for dipole moments $D>0.650 \mathrm{ea}_{0}$, lower than the smallest value $D=0.840 \mathrm{ea}_{0}$ for which Wall is, Herman, and Milne had made calculations.

At the suggestion of $R$. D. Present, we set up the problem in elliptic coordinates. It then became apparent how one might accurately calculate the value of the critical dipole moment. With reference to Fig. 2, one defines the coordinates $\rho=\left(r^{i}+r^{\prime \prime}\right) / R$ and $\mu=\left(r^{\prime}-r^{4}\right) / R$. The rariges of the variaties are given by $1 \leq \rho<\infty$ and $-1 \leq \mu \leq 1$. The Schrödinger equation, 


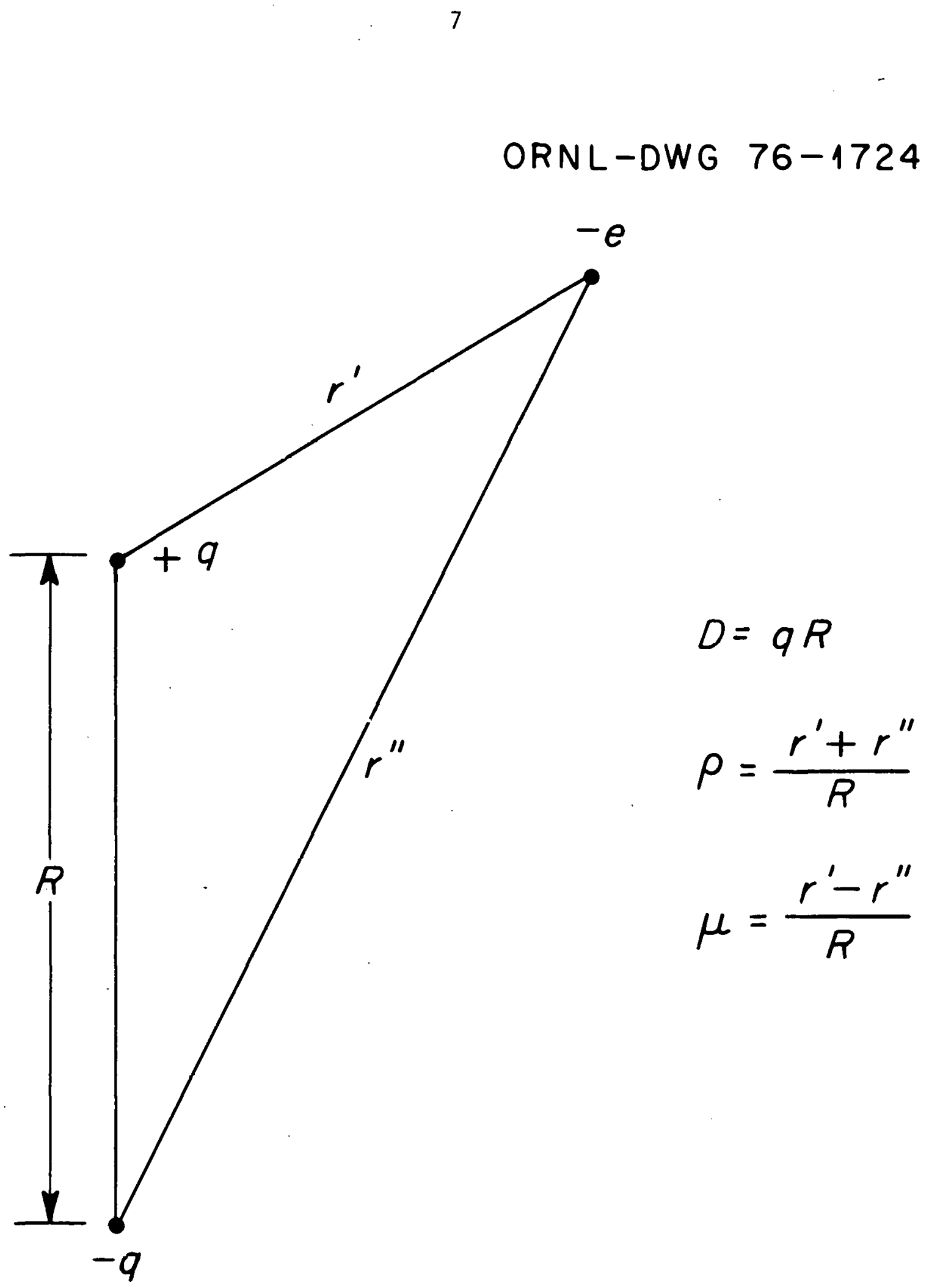

Fig. 2 


$$
H \Psi=\left[-\frac{\hbar^{2}}{2 m} \nabla^{2}+e q\left(-\frac{1}{r^{1}}+\frac{1}{r^{11}}\right)\right] \Psi=E \Psi,
$$

is independent of the azimuthal angle about the dipole axis. In elliptic coordinates with $q=e$, this equation gives

$$
\frac{\partial}{\partial \rho}\left[\left(\rho^{2}-1\right) \frac{\partial \Psi}{\partial \mu}\right]+\frac{\partial}{\partial \mu}\left[\left(1-\mu^{2}\right) \frac{\partial \Psi}{\partial \mu}\right]-\left(\varepsilon \rho^{2}-\varepsilon \mu^{2}+\beta \mu\right) \Psi=0,
$$

where $\varepsilon=-m R^{2} E / 2 \hbar^{2}$ and $\beta=2 R / a_{0}$. The dipole moment appears only in $\beta$, and $i$ ts numerical value in atomic units is given by $R=B / 2$. Fox and $I$ used a trial function in the form of a series

$$
\Psi(\rho, \mu)=e^{-\alpha \rho t / 2} \sum_{p, q=0} C_{p q} \rho^{p} p_{\mu}^{q}=\sum_{p, q=0} C_{p q} \phi_{p q}
$$

in which $\alpha$, $t$, and the $C_{p q}$ are adjustable, real parameters. Picking a fixed value of $R$ and using a finite number of terms from (4), we calculated the expectation value of the Hamiltonian and minimized it by variation of $\alpha$ and $t$. Mathematicalily, one solves for the lowest value of $\lambda$ in the determinatal equation,

$$
\operatorname{det}(H-\lambda N)=0 \text {, }
$$

in which the matrix $\mathrm{N}$ has elements

$$
N_{p q, i j}=\int \psi_{p q} \psi_{i j} d \tau
$$

where $d_{\tau}$ is the volume element. The ratios of the $c_{p q}$ can be calculated for this $\lambda$ from Eq. (5) and used in Eq. (4) to give the normalized wave function. With this variational procedure we eventually got down to a 
binding energy of $8.97 \times 10^{-20} \mathrm{Ry}$, for which a 35 -term trial function gave $D=0.667 \mathrm{ea}_{0} .11$

It was not feasible to go all the way to zero binding energy by the continuation of this procedure because of rapidly increasing computer overflow problems. Instead, it was found possible to re-. arrange the determinant (5) in the limit $t=0$ with $\lambda=0$ in such a way as to factor it into a product of identical, rapidly converging subdeterminants. $^{12}$ The solution to the rank-two sub-determinant gave $D=0.649520$, the same value we found earlier ${ }^{10}$ with the $S$ - and P-state trial function; rank. 3 gave 0.639370; and rank 4, 0.639316. The precise value is $D_{\min }=0.639315 .11,13$

Other Investigators Show Interest

Without giving it much thought one way or the other, I assumed the problem was about wrapped up; but then an unexpected flurry of activity occurred. Fox was in Europe at the time I obtained the final result in the summer of 1966. I wrote up our solution and sent a draft to him on August 19. Incorporating his comments, I submitted a manuscript to Physical Review Letters which was received on September 12. Subsequently, a letter dated September 27 came from Marvin Mittleman, with whom I had had an exchange of correspondence about the dipole problem in August. Mittleman related that he and Myerscough had solved the problem of $D_{\min }$ by using results of an earlier paper of his with von Holdt. 14 Mittleman and von Holdt had found, for a point dipole, that scattering amplitudes could not be obtained for electrons when one of their parameters reached the value 1.279. This number implied that $D-1.279 / 2=0.6395 \mathrm{ca}_{0}$, but they had not interpreted this finding or 
related it to the bound-state problem. Mittleman had received our manuscript to review for Physical Review Letters and suggested in his letter that our two papers, which were entirely different mathematically, be submitted together, but to another journal. He did not feel that rapid publication was necessary and had returned our manuscript to Goudsmit with that recommendation. By this time I was aware that several investigators were following up on papers that Fox and I had published, 90 and so I felt that speed of publication was warranted. We sent the two papers to Physics Letters, where they appear together. ${ }^{12,15}$ Their paper was received on 0ctober 7 and ours on 0ctober 17, editor ter Haar kindly allowing me one re-write to comply with the journal's length restrictions.

On October 21, W. Byers Brown sent me a letter and preprint of the solution that he and Robert $E$. Roberts had obtained for $D_{\min }$. Their solution, which was received by the Journal of Chemical Physics on October 24, was published as a letter. ${ }^{13}$ The October 31 issue of Physical Review Letters contained the abstract of an article with still another solution to be published in Physical Review. This paper, ${ }^{16}$ by Jean-Marc Levy Leblond, was received on August 15, and so he was probably the first of these four groups to find that $D_{\min }=0.639 \mathrm{ea}_{0}$. His method and that of Brown and Roberts are essentially the same. The latter authors also showed that inclusion of a repulsive radial core does not affect the value of $D_{\min }$.

A number of investigations rapidly added to the knowledge of the electron-dipole system. Crawford and Dalgarno ${ }^{17}$ presented a general argument that a non-critical value exists and gave its correct value 
based on the Mittleman-von Holdt paper. ${ }^{14}$ Crawford $^{18}$ showed that the existence of bound states depends only on the value of $D$ when the field is that of either a point dipole plus a sufficiently repulsive core or a finite dipole. Each symmetry class of states has its own critical value of $D$ and an infinite number of states exist when any do. Bound states for the pure point dipole "fall to the center" 19 unless there is a strong repulsive core. Coulson and Walmsley ${ }^{20}$ extended the work of Mittleman and Myerscough and calculated the minimum dipole moments necessary for having excited states with different numbers of nodes and values of angular momentum about the dipole axis.

Possible Physical Implications

Figure 3 summarizes some of the information developed at this point. The solid curve shows the ground-state electronic binding energy calculated by Wallis, Herman, and Milnes as a function of dipole moment. ${ }^{5}$ The dashed curve shows our values. ${ }^{11}$ As seen from the figure, the critical moment falls among values for a number of common molecules. The theory appears to rule out the capture hypothesis introduced to explain the data in Fig. 1. While the dipole moment of $\mathrm{H}_{2} \mathrm{O}$ is sufficient to bind an electron, that of $\mathrm{H}_{2} \mathrm{~S}$ is not. On the other hand, the existence of $D_{\text {min }}$ might be expected to affect scattering behavior in other ways, since additional channels of interaction are opened when $D>D_{\min }$.

An intensive effort was made--without success--during the next few years to detect experimentally some effect to show the existence of $D_{\min }$ in electron-swarm experiments. ${ }^{21-24}$ Theoretical attention shifted ton positive-energy electronic states and to non-stationary 
O.R.N.L. DWG. NO. $66-11753$

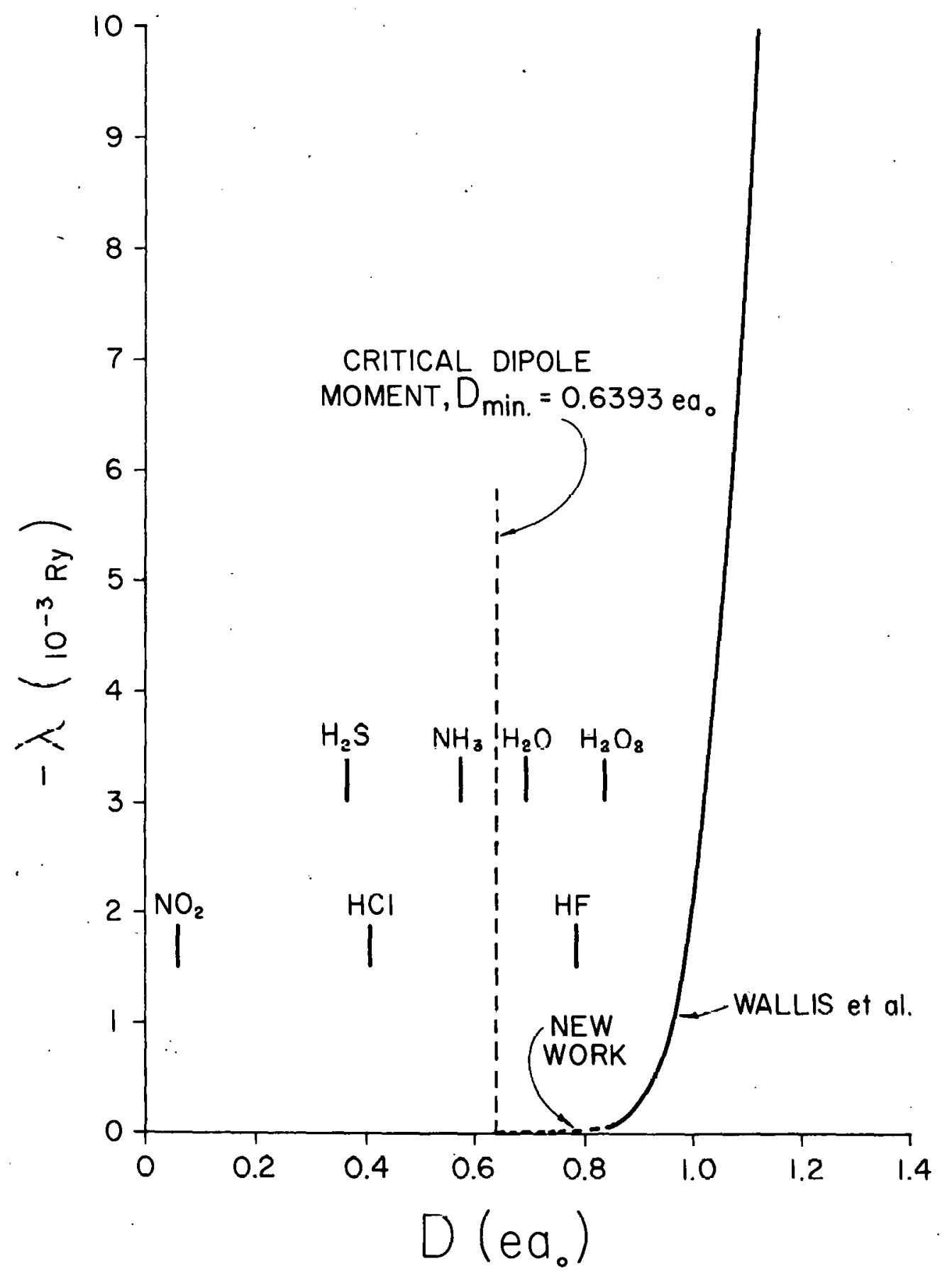

Fig. 3 
dipoles. In early 1969, I was preparing to leave on a year's assignment to CERN in Geneva, Switzerland, and discontinued work on the problem. My colleague, W. R. Garrett, continued the important theoretical investigations at this time. He showed that, for a dipole free to rotate about its fixed center, the critical dipole moment depends on both the moment of intertia I and the length $R$ of the dipole, even for fixed $D$. Moreover, the critical values for rotating dipoles increase with the angular-momentum quantum number. Thus, measurements of drift velocity for an electron swarm in thermal equilibrium with a polar gas at room temperature could not be expected to permit measurement of $D_{\min }$. Garrett's work and the contributions of other investigators are summarized in his 1972 paper. ${ }^{25}$

The Capture of Negative Mesotrons in Matter: Fermi-Teller, 1947

In early 1968 this story took an unexpected turn. At a seminar that Ken Fox gave at the Jet Propulsion Laboratory, where he was working that year, H. B. Snodgrass remarked that he thought the electron-dipole system had been worked out by Wightman in a Princeton thesis in the late 1940's. A search of the Physical Review Index quickly uncovered A. S. Wightman's 1949 paper $^{26}$ entitled "Moderation of Negative Mesons in Hydrogen I: Moderation from High Energies to Capture by an $\mathrm{H}_{2}$ Molecule." Wightman calculated the slowing-down times for negative $\mu$ and $\pi$ mesons and particles with other masses. At high meson velocities ( $v \gg e^{2} / h$ ) he used the ordinary stopping-power formula and at intermediate speeds, an exact Born-approximation evaluation for atomic hydrogen. At low speeds $\left(v<e^{2} / h\right)$ he employed an adiabatic approach in which the negative meson and nuclei are regarded, to a first 
approximation, as being at rest, with the electrons moving in stationary states around them. To understand molecular hydrogen, Wightman first discussed the $\pi^{-}$interaction with atomic hydrogen in some detail. He says, "The qualitative nature of the electron wave function is evident from the fact that for large proton separations, $R$, it must be approximately the wave function of a hydrogen atom, and for small separations that of an electron moving in the potential of an electric dipole of moment eR. For sufficiently small R, such a dipole has no bound states. Consequently, it is clear that there exists a critical radius $R_{C}(0,0)$ such that for $R<R_{C}(0,0)$ no bound states exist. ${ }^{8}$ "Wightman's footnote 8 refers to still earlier work: "... The existence and size of the critical radius for our problem was pointed out by Fermi and Teller, Phys. Rev. $\underline{72}, 406$ (1947)."

The Fermi-Teller paper" 27 is entitled "The Capture of Negative Mesotrons in Matter." They calculate in some detail the slowing down of mesons in the energy range below $2000 \mathrm{eV}$, where the first Born-approximation theory of stopping power is not valid. They show that the time required for a meson to slow down and be captured is short compared with the meson's natural decay time. Fermi and Teller also discuss the influence of the physical and chemical states of matter and the relative probabilities of capture by nuclei of different atomic number in a medium. They derive an energy-loss formula for a particle interacting with a degenerate electron gas and apply it to metals, insulators, gases, and chemical compounds. In discussing insulators Fermi and Teller point out that the energy received by an electron must be at least as large as the gap between two Brillouin zones. Their discussion includes 
the following description: "Furthermore, the actual size of the Brillouin gap is affected by the localization of the mesotron on one lattice atom. Since, at least in the critical cases, the mesotron is captured fairly far inside the atom, the atom is effectively turned into an element with atomic number (z-1). If we are dealing with an isolated atom, this would lower the ionization energy of the atom and turn it in to the much smaller value which usually is called the electron affinity of the atom of charge (2-1). Actually this electron affinity may even be zero. In the special case of mesotron capture by the hydrogen atom, it is found that when the mesotron approaches the nucleus to a distance of 0.639 Bohr radii, the binding energy of the electron becomes zero. In the closed shell structure usually found in insulators ..." The value of the critical dipole moment is thus given in passing, almost as an aside. No other mention is made of $D_{\min }$ in the FermiTeller paper, nor is there any indication of how it was calculated. I had a fear at this point that the calculation might be trivial. At any rate, unknown to the four groups $12,13,15,16$ who were in press simultaneously to publish $D_{\min }$, and unknown to a number of authors of subsequent papers-and journal reviewers--this discovery was made independently in molecular physics some twenty years after it had been made in particle physics. If the Fermi-Teller calculation was so simple that the method was not worth noting, then that fact, too, had escaped the molecular theorists.

I tried to think of ways to uncover the Fermi-Teller solution. The first thing I did was consult the University of Chicago Press two volumes of Fermi's Collected Papers, ${ }^{28}$ which have introductory notes to many of his publications. The paper in question is No. 233, and is 
introduced by Teller together with paper No. 232, entitled "The Decay of Negative Mesons in Matter." Teller's introduction is included here:

$$
N^{0} 232 \text { and } 233 .
$$

Fermi's interest in the peculiarly small interaction of the $\mu$ mesons with nuclear matter was excited by the work of M. Conversi, E. Pancini and 0. Piccioni. In a colloquium at which Fermi was absent, I happened to hear about this work. A few days later I told Fermi about the puzzling fact found by the Italian group that decay of negative 1 mesons seems more probable than reaction with the nuclear matter in the carbon nucleus. It was at once obvious that this points toward a very weak interaction of $\mu$ mesons and nucleons. Weisskopf had arrived at a similar conclusion and through correspondence we arranged a short joint note.

At the same time Fermi felt that alternative explanations should be investigated as vigorously as possible. He wondered whether the process of capturing the $\mu$ meson into its lowest orbit around the carbon nucleus may perhaps take a considerable length of time. If so, the relatively great number of $\mu$ meson decays might take place during the time of capture. As a result we looked into the capture process in very great detail. Actually in our conversations I was most of the time the listener. Due to the fact that the statistical (FermiThomas) picture of the atom proved adequate, the answer could be obtained in a straightforward and convincing way. There was only one somewhat difficult question: Whether a relatively high number of $u$ mesons could be trapped in high angular momentum states around the nucleus. (Energy loss from these states is more difficult.) In the end an orderly survey of the energy loss mechanisms in varying substances proved feasible.

At the time when this article was written Fermi and I shared a secretary (Nancy McMillan) who had a degree in chemistry. The paper was actually written by giving dictation to Mrs. McMillan. Since Fermi and I frequently interrupted each other in the actual formulation the first draft became a little confused. That a draft was produced at all was due to the excellent work of our secretary. It was then my job to transform the first draft into a manuscript, The Copture of Negative Mesotrons in Matter. This I did (I am afraid) with limited success.

$$
\text { E. Teller. }
$$

No mention of the critical charge separation for electronic binding. 
Teller visits ORNL

It was about this time that I learned that Edward Teller was coming to ORNL to visit the Civil Defense project. His schedule in Oak Ridge was extremely tight, but I hoped to have an opportunity to at least ask him about the 1947 paper. The program for Friday afternoon, April 26, 1968, called for Karl Morgan, Director of the Health Physics Division, to drive Teller from the $X-10$ site of ORNL over to the Biology Division at Y-12--about a ten-minute trip. Morgan graciously consented to my request to accompany the two of them and use this opportunity to ask Teller about the calculation of $D_{\min }$.

During the short time available we only briefly got into the problem. Teller did not recall off hand how the number was arrived at for the 1947 paper, but made one or two suggestions and said he would write me after looking into his files when he returned to Livermore. In May I wrote him that I was unable to carry through a derivation based on our conversation. On October 22 Teller wrote back, "At last I have gotten around to considering the dipole problem which we discussed. I am quite sure that at the time Fermi and I worked together I handled this portion by using the separate two-center equation and used some simple approximation, I forget which." He went on to describe a handbook article he was preparing with H. L. Sahlin on two-centeir problems. ${ }^{29}$ In this paper Teller and Sahlin used the separated equations and employ a variational principle to obtain upper bounds for pairs of values of $R$ and the separation constant. They obtain a very simple approximate solution, which yields $D=0.6394$ ea $_{0}$ for zero energy. 
With this development the historical quest of the problem appeared to be finished without having uncovered the 1947 solution.

Four Years Later--Lunch at the University of Chicago

It was not until January of 1972 that I got excited again about the possibility of tracking down the original calculation of the critical dipole moment. Ray Garrett and I had visited Argonne National Laboratory and, before leaving for Oak Ridge, we had lunch at the Universty of Chicago with Ugo Fano and several of his graduate students. The discussion got around to our describing the history of $D_{\min }$ as related above. Fano observed that Fermi's collected papers and notebooks are located there on the campus in the University Library. Armed with the information that the Fermi-Teller paper was received by the Physical Review on May 28, 1947, one of the students, Ted Roth, wrote me shortly thereafter that he had found about fifteen notebook pages on the dipole problem in the Fermi collection. The work showed a number of attempted solutions and several numerical integrations involving hyperbolic functions. The material appears in Notebooks 100 (D12), covering the period October 5, 1945-January 7, 1947, and 101 (D11), covering January 8, 1947-June 12, 1947. Roth arranged for the Library to send me photocopies of these pages. The following material and photographs are made possible by the Curator of Special Collections, University of Chicago Library.

Fermi's Notebooks $100(012)$ and 101 (D11)

Figure 4 shows the beginning of Fermi's calculation on page 285, dated December 31, 1946. of Notebook 100 (D12). On this page and the 
19

Dee 311946

Orbit of electron in field of $a+t$ $+2 \quad-e$ and a $-e$ charge at fixed de stance

Hleptical coordinates

$$
\begin{aligned}
& \sum_{+2 b^{\prime}} \\
& r^{\prime}=b(\rho+\mu) \\
& r^{\prime}+r^{\prime \prime}=2 k \rho \\
& \rho>i^{\prime \prime}=b(\rho-\mu) \\
& r^{\prime}-r^{\prime \prime}=26 \mu \\
& -1 \leq \mu \leq 1 \\
& \Delta f=\frac{1}{t_{\theta^{2}}\left(\rho^{2}-\mu^{2}\right)}\left[\frac{\partial}{\partial \rho}\left\{\left(\rho^{2}-1\right) \frac{\partial f}{\partial \rho}\right\}+\frac{\partial}{\partial \mu}\left\{\left(1-\mu^{2}\right) \frac{\partial f}{\partial \mu}\right\}+\right. \\
& \left.+\left(\frac{1}{\rho^{2}-1}+\frac{1}{1-\mu^{2}}\right) \frac{\partial^{2} f}{\partial \varphi^{2}}\right] \\
& \text { for } \sum \text {-states } \\
& \Delta \psi+\frac{2 \mu m}{\hbar^{2}}\left(E+\frac{e^{2}}{r^{\prime}}-\frac{e^{2}}{r^{\prime \prime}}\right) \psi=0 \\
& {\left[\left(e^{2}-\rho \psi t\right)\left[\frac{\partial}{\partial \rho \rho}\left\{\left(\rho^{2}-1\right) \frac{\partial \psi}{\partial \rho}\right\}+\frac{\partial}{\partial \mu}\left\{\left(1-\mu^{2}\right) \frac{\partial \psi}{\partial \mu}\right\}\right]+\right.} \\
& +\frac{2 m b^{2}}{\hbar^{2}}\left(E^{\left(\beta^{2}-\mu^{2}\right)^{2}(\gamma-\mu)}-\frac{e^{2}\left(\varphi^{2}+\mu\right)}{2}\right) \psi=0
\end{aligned}
$$

Fig. 4 
next, Fig. 5, he introduces atomic units and separates the twodimensional schrödinger equation in elliptic coordinates $\rho$ and $\mu$. Equation (3), above, with $\psi=u(\rho) \vee(\mu)$ leads directly to the two differential equations in the lower half of Fig. 5 , in which $\alpha$ is the separation constant. On the next page, which is dated January 3, 1947, Fermi introduces the transformation $\rho=\operatorname{coth} x$ and obtains for the "radial" equation

$$
\frac{d^{2} u}{d x^{2}}=\left(\frac{\alpha}{\sinh ^{2} x}+\varepsilon \frac{\cosh ^{2} x}{\sinh ^{4} x}\right) u,
$$

where $0<x<\infty$. The substitution $\alpha=-\varepsilon-\gamma$ leads to

$$
\frac{d^{2} u}{d x^{2}}=\left(\frac{\varepsilon}{\sinh ^{4} x}-\frac{\gamma}{\sinh ^{2} x}\right) u,
$$

in terms of the modified separation constant $\gamma$. In the "angular" equation Fermi introduces a new variable by writing $\mu=\tanh z$, with $. \infty<z<\infty$. The second of the separated equations in Fig. 5 then becomes, in terms of $z$,

$$
\frac{d^{2} v}{d z^{2}}=\left(\frac{\varepsilon}{\cosh ^{4} z}+\frac{y}{\cosh ^{2} z}+\beta \frac{\sinh z}{\cosh ^{3} z}\right) v,
$$

which appears on page 293 of the Notebook.

The next severai pages in Notebook 100 (DI2) are taken up with trial solutions, numerical approximations and sketches of various functions and terms, particularly for small $\varepsilon, \gamma$, and $x$. On page 297, which appears as Fig. 6, he solves the radial equation for zero energy and small $x$, obtaining the condition $\gamma<1 / 4$. This is the last page in Notebook 100 (D12). 
21

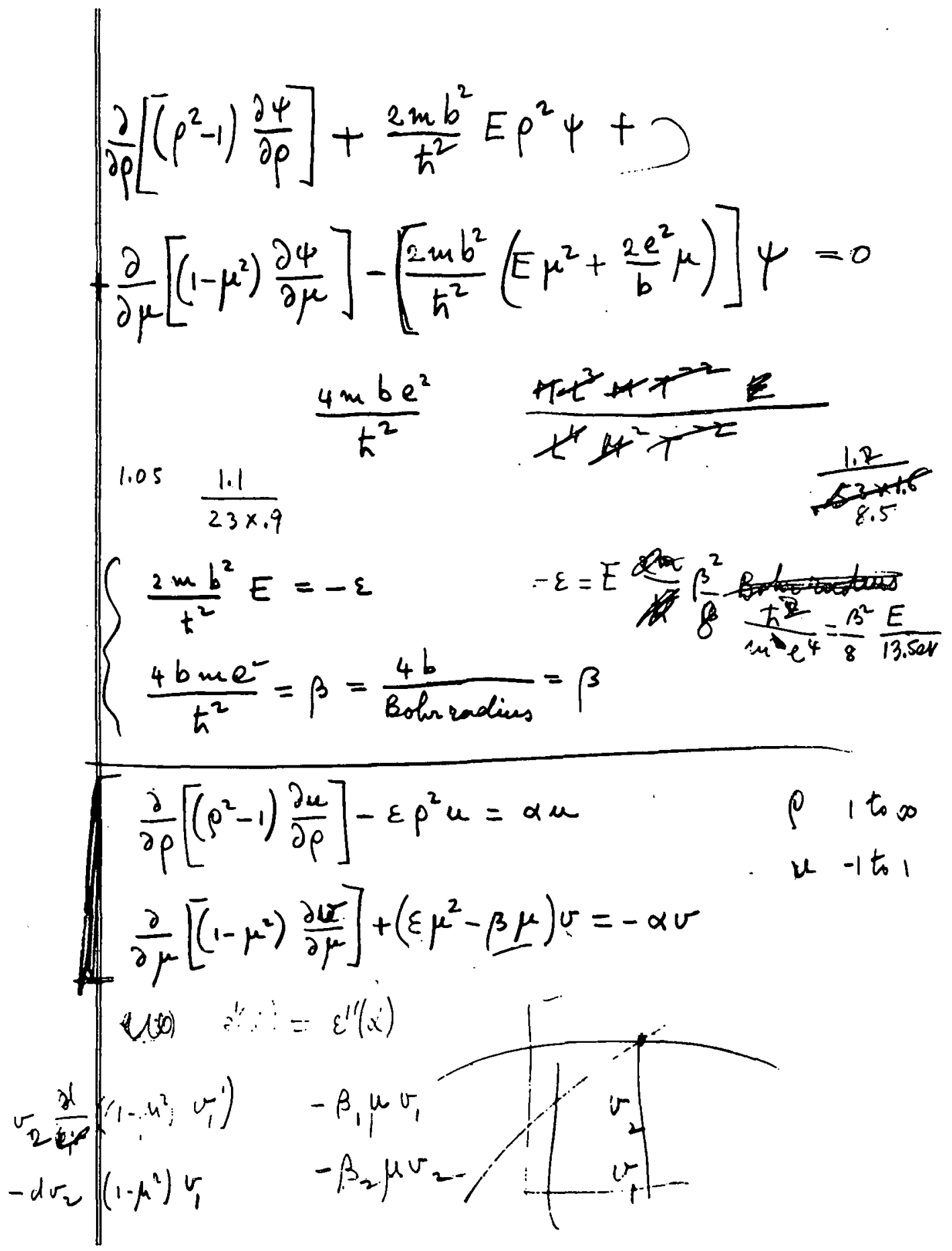

Fig. 5 
22

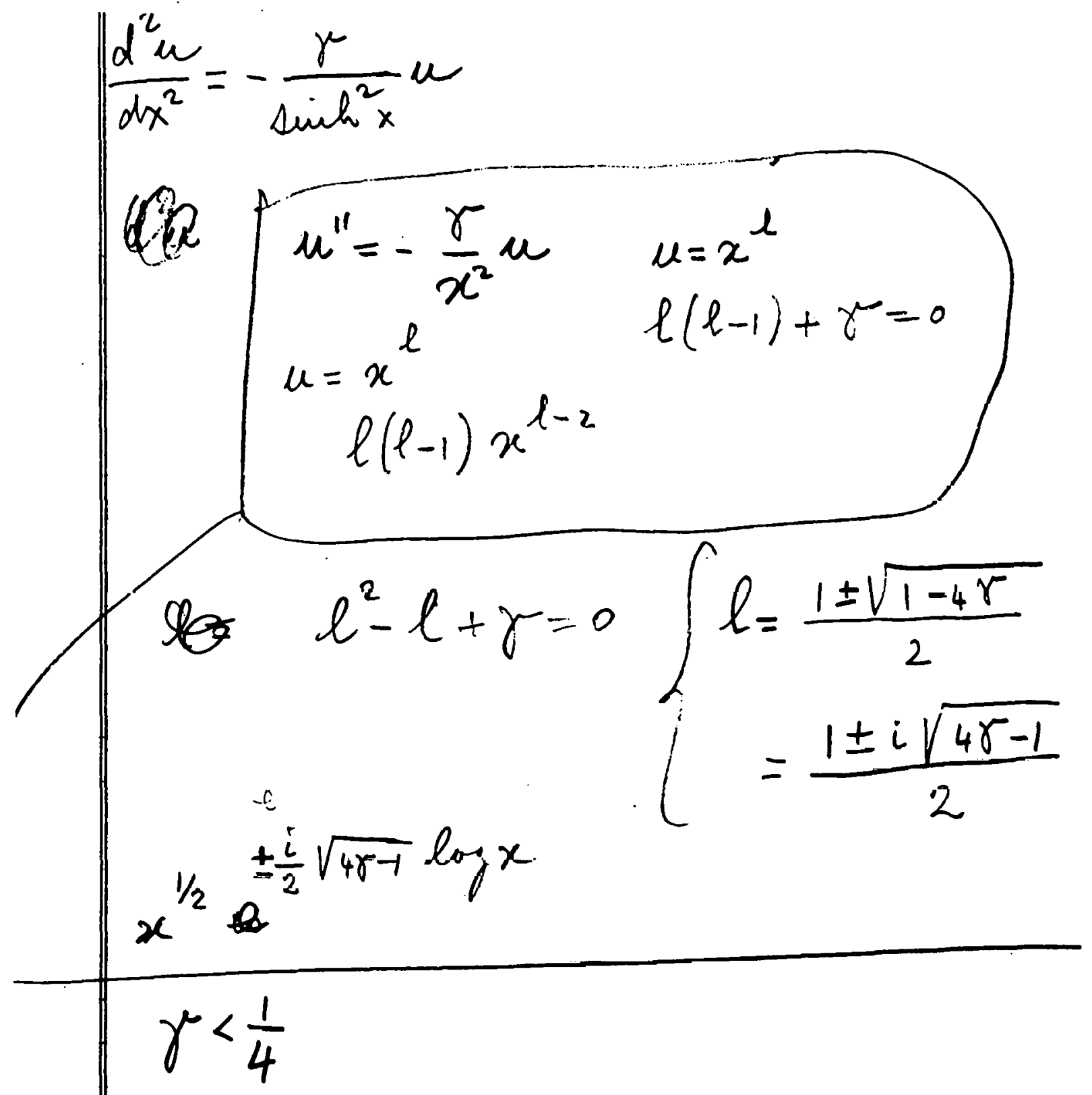

Fig. 6 
Fermi continues the dipole problem by rewriting the separated equations on page 3 of Notebook 101 (011), except that he changes the separation constant from $\alpha$ to $-\alpha$ and writes, now, $\alpha=\varepsilon+\gamma$. Taped at right angles to page 3 along the center margin of the notebook is a single sheet of "Explanations added 2/10/50" (some three years later). The two sides of this insertion are shown in Figs. 7 and $8 .{ }^{30}$ At the bottom of the front side, Fermi summarizes the derivation of the separated equation, which he had written on page 3 of Notebook 101 (D11). On the back of the sheet, Fig. 8, he indicates that, when $\varepsilon=0$, the first [radial] equation gives $\alpha=1 / 4$. (With reference to Fig. $6, \alpha=\gamma$ when $\varepsilon=0$.$) The second [angular] equation determines the critical value of$ b. He references page 12 for the numerical solution.

Following these inserted explanations, pages 4-6 in Notebook 101 (D11) contain numerical calculations, using expansions for the powers of sinh and trying a solution of the form $u=e^{-f}$. At the hot.tom of page 6 is written the last date, January 9, 1947. Pages 7 and 8 have a rough numerical solution for $\gamma=0.09$ and $\varepsilon=0$, based on the fact that $u$ is constant for large $x$. On page 9 Fermi returns to $u=e^{-f}$ with $x$ smal1, and on page 10 he calculates $1 / x^{2}, 1 / x^{4}-1 / x^{2}$ and $\gamma\left(1 / x^{4}-1 / x^{2}\right)$ with $x$ varying between 0 and 3.0 in steps of 0.1 .

Page 11 , shown in Fig. 9, gives a numerical solution of the radial equation (8) with $\varepsilon=0$ and $\gamma=1 / 4$. In the original wave equation (Fig. 4), $\rho$ varies between 1 and $\infty, \rho=1$ representing the line connecting the two charges. The transformation $\rho=\operatorname{coth} x$ inverts the coordinates so that, when $x$ tends toward infinity, $\rho$ tends toward unity. Thus, when $x$ is large the electron is near the dipole and the radial wave 
24

Explanations added $2 / 10 / 50$

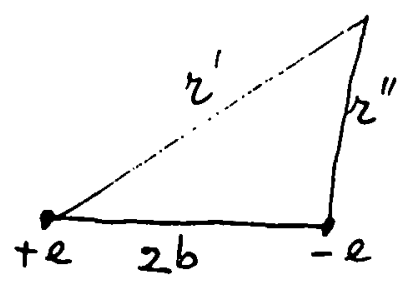

(See MG 3)

$r^{\prime}+r^{\prime \prime}=26 \rho \quad \frac{904}{\rho} \geqslant 1$

$r^{\prime}-r^{\prime \prime}=2 b \mu \quad-1 \leq \mu \leq 1$

$\varphi=$ azimuth

$$
\begin{aligned}
\nabla^{2} \psi=\frac{1}{b^{2}\left(p^{2}-\mu^{2}\right)} & {\left[\frac{\partial}{\partial p}\left\{\left(\rho^{2}-1\right) \frac{\partial \psi}{\partial p}\right\}+\frac{\partial}{\partial \mu}\left\{\left(1-\mu^{2}\right) \frac{\partial \psi}{\partial \mu}\right\}+\right.} \\
& \left.+\left(\frac{1}{p^{2}-1}+\frac{1}{1-\mu^{2}}\right) \frac{\partial^{2} \psi}{\partial \varphi^{2}}\right]
\end{aligned}
$$

Schrodinger equations for $\psi$ iedep. of $\varphi$

$$
\begin{aligned}
& \frac{1}{b^{2}\left(\rho^{2}-\mu^{2}\right)}\left\{\frac{\partial}{\partial \rho}\left[\left(\rho^{2}-1\right) \frac{\partial \psi}{\partial \rho}\right]+\frac{\partial}{\partial \mu}\left[\left(1-\mu^{2}\right) \frac{\partial \psi}{\partial \mu}\right]+\right. \\
& +\frac{2 m}{\hbar^{2}}\left[E+\frac{e^{2}}{b} \frac{1}{\rho+\mu}-\frac{e^{2}}{b} \frac{1}{\rho-\mu}\right] \psi-0
\end{aligned}
$$

Put $\beta=\frac{4 b}{a}$ with $a=\frac{\hbar^{2}}{m c^{2}}=$ Bohr radius

$$
\varepsilon=-\frac{2 m b^{2}}{\hbar^{2}} E
$$

$$
\frac{\partial}{\partial \rho}\left[\left(\rho^{2}-1\right) \frac{\partial \psi}{\partial \rho}\right]+\frac{\partial}{\partial \mu}\left[\left(1-\mu^{2}\right) \frac{\partial \psi}{\partial \mu}\right]=\left(-\varepsilon \rho^{2}+\varepsilon \mu^{2}+\beta \mu\right) \psi
$$

Separates with $\psi=u(\rho) v(\mu)$ and $\alpha=$ arbitrary bust eq into equations on $p .3$

Fig. 7 
25

Look for $b$-that gives $\varepsilon=0$ because at this value of $b$ electron will bread loose

It seems that first equation then setermues $\alpha=1 / 4$ \& second equation determine b. (for numerical solution see p.12)

Equation for $v$ becomes

$$
\frac{d}{d \mu}\left(\left(1-\mu^{2}\right) \frac{d v}{d \mu}\right)=\left(\beta \mu+\frac{1}{4}\right) v
$$

This transforms by $\mu=\operatorname{tgh} x \quad-\infty<x<\infty$

$$
\frac{d^{2} v}{d x^{2}}=\left(\frac{1}{4}+\beta \operatorname{tgh} x\right) \frac{v}{\cosh ^{2} x}
$$

For $\beta>1 / 4$ coff of $\sigma$ plats

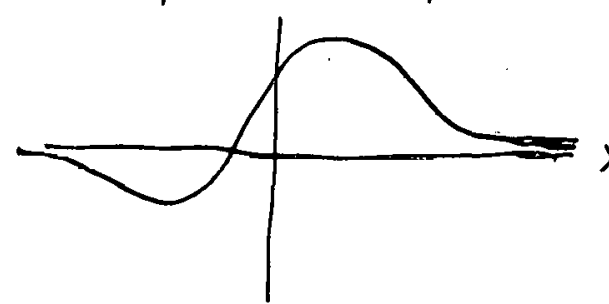

$x$ It presumably has an e.v. $\beta$ for which v plots

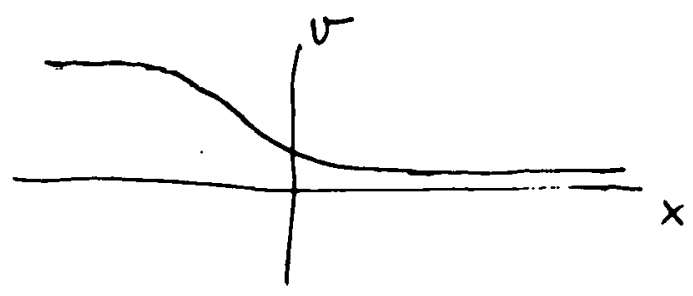

Fig. 8 
26

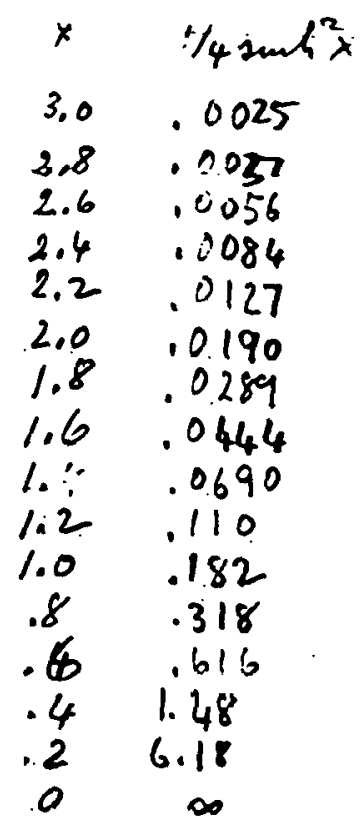

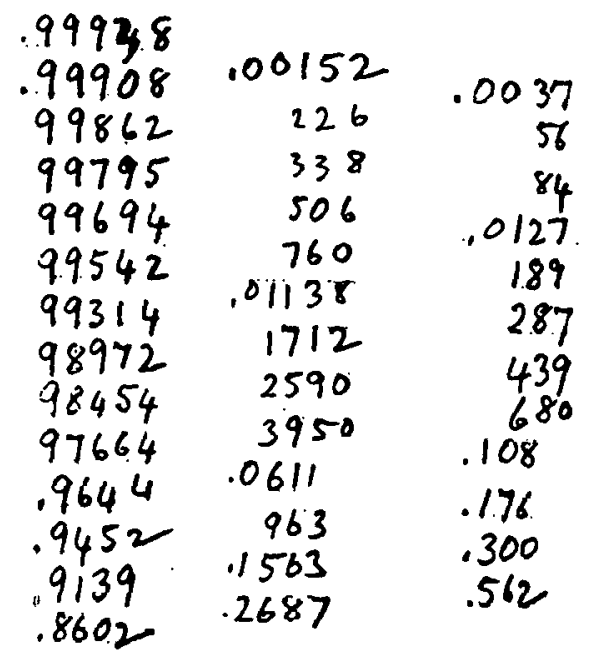

.9635

$$
y^{\prime \prime}+\frac{1 / 4}{\sinh ^{2} x} y=0
$$

$y^{\prime}=\frac{1}{4} \int_{x}^{\infty} \frac{y d x}{\sinh ^{2}} \approx \frac{1}{4}(\operatorname{coth} x-1)$

cy $y \approx \operatorname{const}+\frac{1}{4}(\log (\sinh x)-x)$

$$
\begin{gathered}
1=\frac{1}{4}\left(\log \left(2 e^{x}\right)-x\right)+c \\
y \approx \frac{1}{4}(4-x+\log (2 \sinh x)) \\
1.17520
\end{gathered}
$$

Fia. 9 
function, which Fermi denotes by $y$ on page 11 , does not change much as $x$ approaches infinity. The condition $y$ \& constant at large $x$ permits integration of the radial equation in closed form. With the boundary condition $y(\infty)=1$, the approximate solution for large $x$ is given at the bottom of page 11. The formulas can be used to calcuate $y$ and $y^{\prime}$ at large $x$ and begin the numerical integration of the radial equation toward $x=0$. The last three columns give $y, y^{\prime}$, and $y^{\prime \prime}$, starting from the values calcuated for. $y(3.0)$ and $y^{\prime}(2.9)$ with $\Delta x=0.2$. It is clear that $x=3$ is "large," because $\rho=\operatorname{coth} 3=1.0050$, showing that the electron coordinate is almost on the dipole axis. On page 12 (Fig. 10) Fermi integrates over a fine mesh, $\Delta x=0.02$, from $x=1.00$ to zero. The starting entries in columns 3 and 4 are $y(1.00)$ and $y^{\prime}$ (0.99), calculated from the formulas on page 11.

From the description in Fig. 8 I assumed that the numerical solution for the critical value of $b$ appears on page 12. At first glance it is not altogether obvious exactly what the numbers represent. Toward the bottom of page 12 there appears to be an interpolation around the value 0.6234 , which catches the eye. Detailed work shows, however, that the last three columns on page 12 represent the same quantities as they do on page 11: $y, y^{\prime}$, and $y^{\prime \prime}$, but over a finer mesh and over the smaller interval, 1 to 0.

Fermi began a new problem on page 13, and nothing more on the dipole appears in the Notebook, which covers the period beyond receipt of the 1947 manuscript by the Physical Review. I searched the notes described here high and low for any clue as to how Fermi determined the critical value of b. I reproduced virtually every 


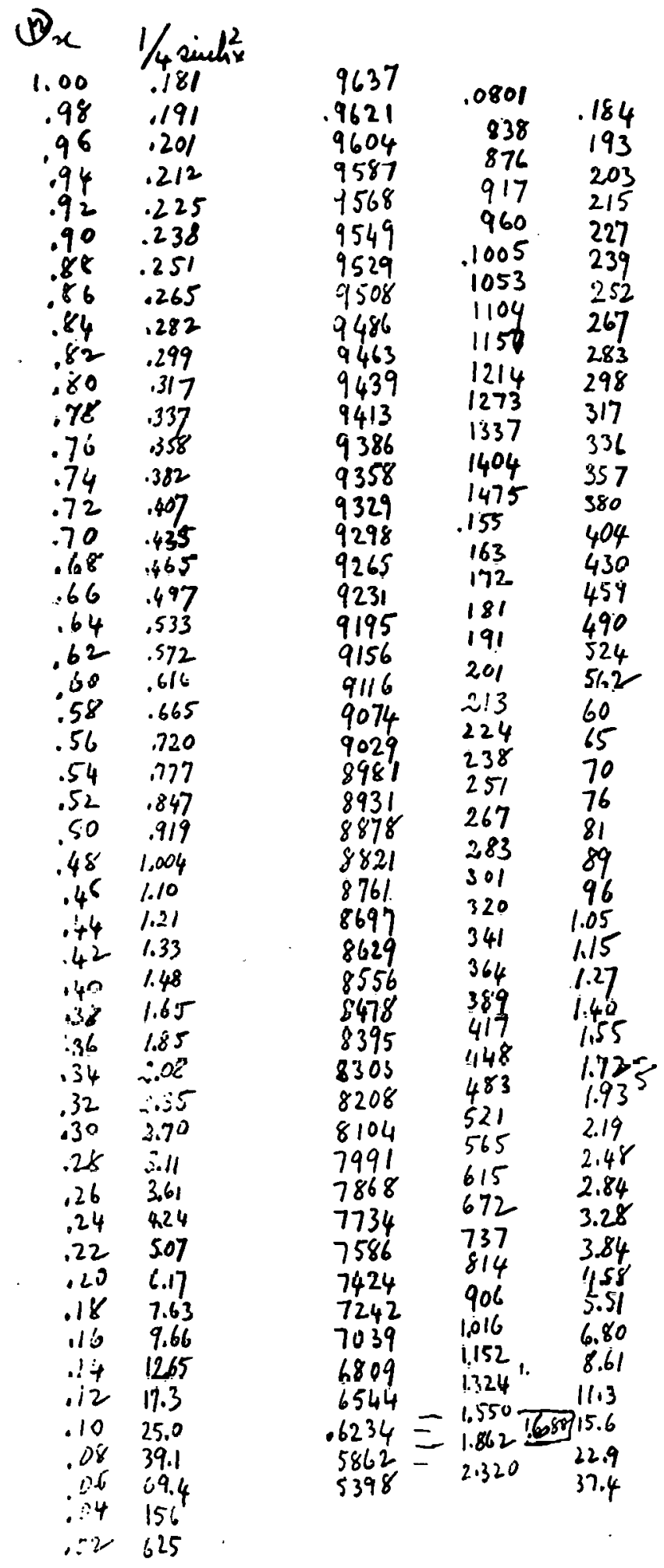

Fig. 10 
number he had written in various places. In Fig. 9, for example, $1.17520=\sinh 1 ; 3.854=3+\ln 2 \sinh 1=4 y(1)=4 x .9635$. Ray Garrett and Hal Schweinler went through the notes with me several times. After much work, I gave up again.

A Visit to the Fermi Collection, June 1975

I could never quite get the problem out of my mind, though. I intended to visit the Fermi collection someday and have a firsthand look. Considerable time had passed when I received a letter in the spring of 1975 from Fano about another matter. In answering him, I decided that there would never be a better time to visit Fermi's collection; and so I asked Fano to put me in touch with the proper authorities. I boned up again on the mathematical details of Fermi's notes and spent June 24 at the Regenstein Library, University of Chicago.

When you visit the Fermi collection, you are shown a list that briefly describes the collection and, in some detail, the contents of the 49 boxes that comprise it. The listing is not indexed, and so some study is required to get a bearing on where specific matcrial might be located. The photocopies that I had from Ted Roth came from the Notebooks in Box XX of the collection, and so I started there. Figure 11 shows the reading room in the Special collections Department of the Regenstein Library, where material is brought to visitors for study. From the windows of the reading room, one can see the monument that marks the site of the first chain-reacting pile on December 2, 1942. 


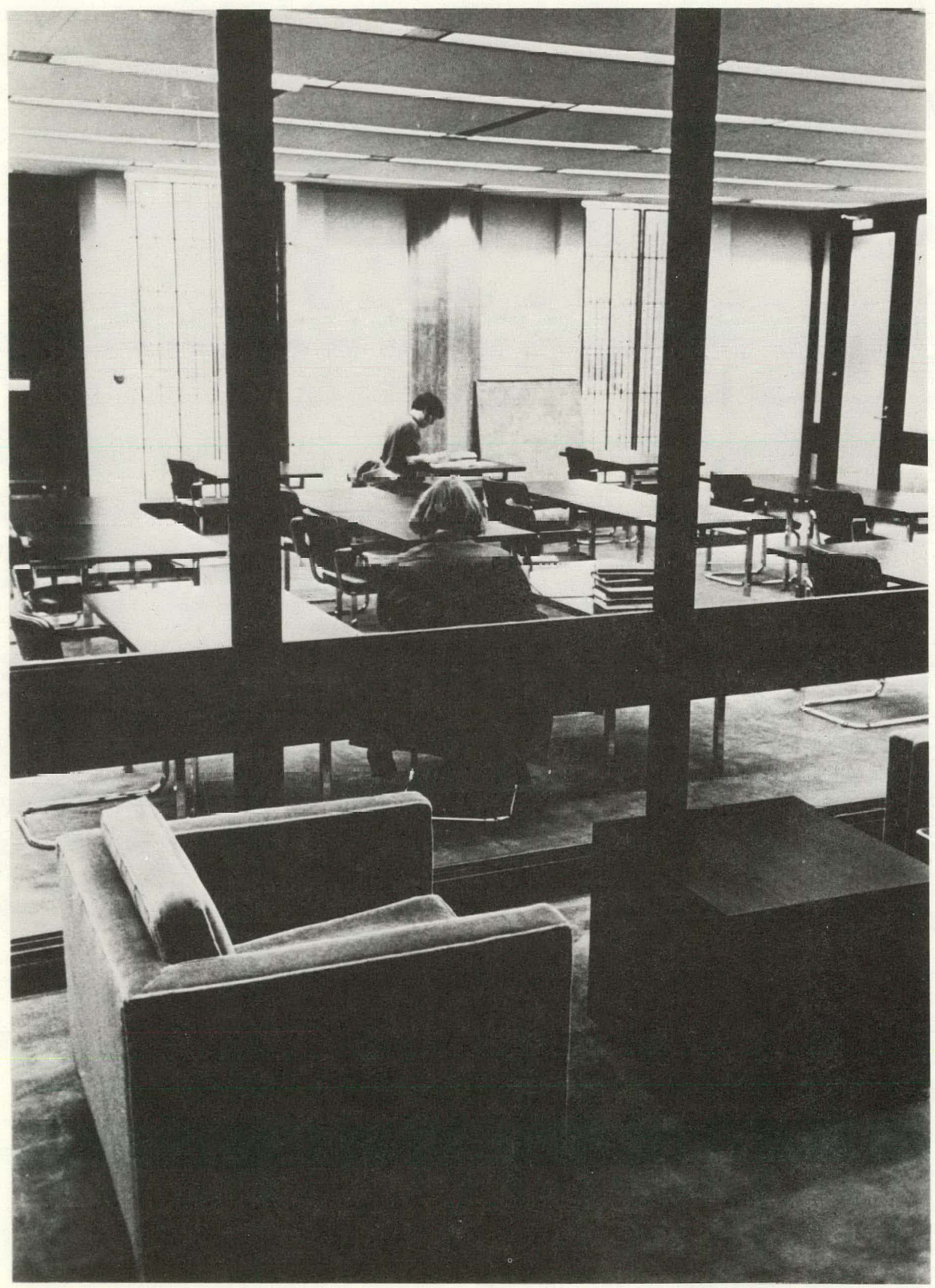

Fig. 11 
I looked through Notebooks 100 (D12) and 101 (D11) page by page to try to find any calculations Fermi had made on the dipole problem in addition to those I already had. I ended the day no more enlightened than I had begun it, except for having clarified one mystery. The upper right-hand corner of the "Explanations added" sheet (Fig. 7) bears the notation "See MG 3/904." I felt that this reference could be a key to the missing calculation of $D_{\min }$. I searched without success through several boxes that contain Fermi's "Artificial Memory." Mary Janzen Wilson of the Library's staff, who was helping me look through some folders in another box, found a sheet of paper bearing the label "MG 3/903." It contained, in Fermi's handwriting, the expressions for the square of the element of distance, the element of volume, and the Laplacian in parabolic coordinates. Behind it was "MG 3/904" with these quantities written in elliptic coordinates (Fig. 12).

When I returned to 0ak Ridge I went through the numerical calculations again to try to see whether I had missed something essential. I went through page 12 (Fig. 10) of Notebook 101 (DI1) once more in detail, especially over the region around $x=0.10$, where Fermi had apparently interpolated. I used a mesh $\Delta x=0.002$ and plotted the functions $y, y^{\prime}$ and $y^{\prime \prime}$ in an attempt to discover any evidence of peculiar behavior there. R. N. Hamm and H. A. Wright joined in this search, but nothing new turned up. The reason for the special markings near the bottom of page 12 remains a mystery to me. During the next few weeks, as I continued looking through the notes I had, I thought of three other, more remote, possibilities for finding the solution. First, Fermi may have worked out $D_{\text {min }}$ 


$$
\begin{aligned}
& \text { Uliptical rasdinates zotatizal! } \frac{1193}{904} \text {. } \\
& \underset{2 a \rightarrow i^{\prime \prime}}{i^{\prime}, r^{\prime \prime}} \begin{array}{l}
\text { freal rodii } \\
2 a
\end{array} \\
& r^{\prime}+r^{\prime \prime}=2 a \rho \quad \rho>1 \\
& r^{\prime}-z^{\prime \prime}=2 a \mu, \quad-1 \leq k \leq 1 \\
& \varphi=\operatorname{arinuth} \theta \quad 0 \text { osic } 2 \pi \\
& \left.d s^{2}=a^{2} \Gamma \frac{p^{2}-u^{2}}{\rho^{2}-1} d p^{2}+\frac{p^{2}-u^{2}}{1-u^{2}} d u^{2}+\left(\rho^{2}-1\right)\left(1-\mu^{2}\right) d \varphi^{2}\right] \\
& d \tau=a^{3}\left(p^{2}-u^{2}\right), d \rho d u d \rho \\
& \left.\left.\left.\Delta \dot{f}=\frac{1}{a^{2}\left(p^{2}-u^{4}\right)}-\frac{\partial}{\partial p}\left\{\left(p^{2}-1\right) \frac{\partial f}{\partial \rho}\right\}+\frac{\partial}{\partial u}\left(1-u^{2}\right) \frac{\partial f}{\partial u}\right\}+\frac{1}{\left(p^{2}-1\right.}+\frac{1}{\left(-u^{2}\right.}\right) \frac{\partial^{2} f}{\partial p^{2}}\right\}
\end{aligned}
$$

Fig. 12 
after the 1947 article was submitted in May and inserted its value in proof. Therefore, I should search his notebooks through the end of 1947, by which time the proofs were probably returned to the Physical Review. Second, I should try to find what he was working on around February 10, 1950, the date of the added explanations. Something on that day led him to add the sheet to Notebook 101 (D11). Third, I could see whether the Fermi collection had copies of galley proofs or correspondence with the Physical Review which could possibly enlighten

me. ${ }^{31}$ Perhaps the Physical Review still had a file on the paper. ${ }^{32}$

Second Visit, October 1975

On October 7 I returned to the Regenstein Library with limited optimism, feeling that this would probably be the last chance to find Fermi's solution. I first looked through successive Notebooks, page by page, through the spring of 1948. The first plan failed. There was no evidence of further work on the dipole problem. Next, in Box XIX I located Notebook 107 (D5), covering the period from December 24, 1949 to May 3, 1950. Fermi wrote an index in the back pages of each Notebook, and so I first turned there. Figure 13 shows this index, which is typical in illustrating the range of problems treated in his notes. What caught my eye at once, of course, was the entry, "Meson capture by hydrogen 54-57." Page 54 is shown in Fig. 14. Taped to the bottom half of this page is an overlay sheet, which is folded out in the figure. Page 54 bears the date February 10, 1950 and shows the long sought calculation of the eigenvalue $\beta=1.28$. 
34

Ind2x

? $\beta$-transitions of nucher inlits $1-16,20,22-23,46-49$

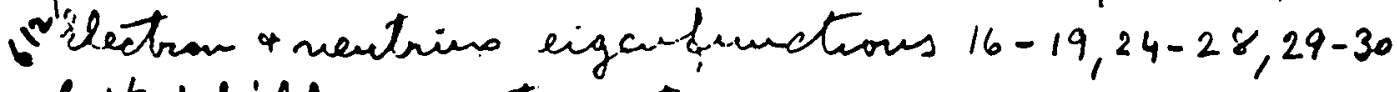
vovitchfield po-uteraction 21 3 Dirac untrices $29,31,36$

vForbidden $\beta$ - spectron 31-34

iv Terbolane a Magreto hydrodyuamies 35

* Pions as two mileon systews 37-43

* Ra $E-\beta$ spectrum 44

${ }^{2}$ Gaunow fuctor of $N^{\prime 5^{*}} \rightarrow C^{14}+H^{\prime}+.5 \mathrm{MeV} 45$

is Resonance effect on statistical atom model. 50-51

Heson decay from Bohr-life orlit 52-53

4. Meson capture in idrom 54-57

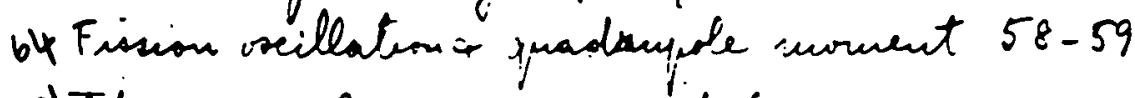

601 Thee uncleons in $p$ - ilatk 60-61.

$6^{11}$ Two mucleons in $p$-ahell $62-65$

$6^{3}$ Range of muchen ferses 67-70

$60^{2}$ Eigene ficastimes of. Re $91-73$

${ }^{0^{2}} \mathrm{E}$.f of $\mathrm{C}^{10}+\mathrm{B}^{10} 74$

$6^{02} E$.f of $\mathrm{Be}^{8}, \mathrm{Li}^{2} 75-79$

60 The $7 / 2$ - 2hall 80

915 Expanding universe (Stiady stete thery) $79-83$ 12 Conchaterus in cartrope Turbolence 84-85 203 Data on light house tube ML-2C39A, 85

$3{ }^{6}$ Klem . Lezdin eq. in e.m. C.uld 86-87

346 Reflection properties of sijuen: 88-89

(0) Dinentron 90

Fig. 13 


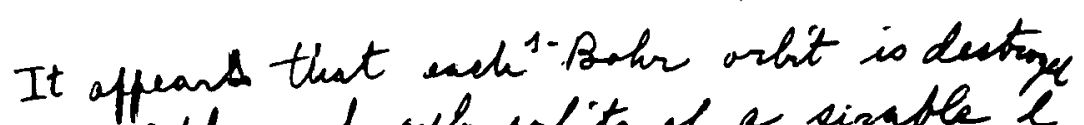
very quickly and only orbits of a sizable $l$ survive. Rate limited by transitions (radiative or otherwise) into orbs of

$\frac{\text { lower l }}{\text { Capture of mesons by A (See } 101 \text { p } 3 \text { ) }}$

r.vio of equation
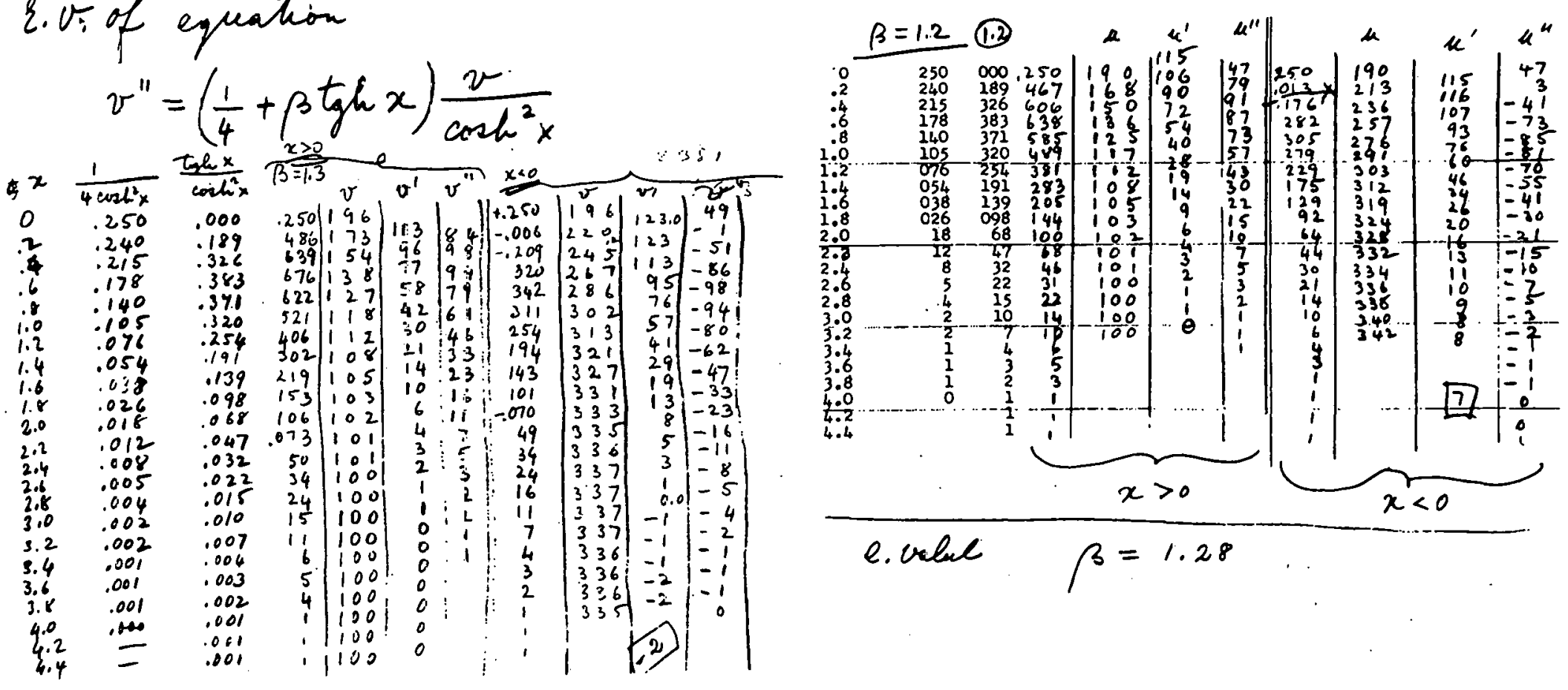

w

Fig. 14 
Fermi references the separated equations in Notebook 101 (DI1), page 3 . The left-hand table gives the calculation for $\beta=1.3$; the right-hand, for $\beta=1.2$.

As seen from Fig. 8, the wave function is constant for large, positive $x$. Fermi evidently calculated the coefficient $v$ out to $x=$ 4.4, where it is almost zero, and started the numerical integration at the bottom of the table for $x>0$ by setting $v(4.4)=100$ and $v^{\prime}(4.3)=0$. The numbers on the left-hand side of Fig. 14 then follow. The term with $\tanh x$ changes sign when' $x$ becomes negative. The integration is continued out to $x=-4$, where the first derivative becomes constant, $v^{\prime}=-2$. The process is repeated with $\beta=1.2$ in the right-hand table, where the first derivative is found to approach the value $v^{\prime}=7$ for large negative $x$. Linear interpolation implies that the first derivative would approach zero when $\beta=1.28$. Fermi goes on for the next two-plus pages to consider the cross section $\left(3.6 \times 10^{-17} \mathrm{~cm}^{2}\right)$ for this process. He estimates that a $3000 \mathrm{eV}$ IIIsun wuld experfence 71 collisions within the critical distance in monatomic hydrogen before coming to rest.

While Fermi's solution at last turned up, it still leaves some questions unanswered. There must have been some work done elsewhere--or still lying undiscovered in one of the 49 boxes of the Fermi collection--leading to the knowledge that the critical $\beta$ is between 1.3 and 1.2. The first three columns on the taped-in right-hand side of Fig. 14 are typed, suggesting that they are copied from notes elsewhere. 
In summary, I cannot say exactly how $D_{\text {min }}$ was first calculated. That it is not in Fermi's 1947 Notebooks is consistent with Teller's recollection of having done that part of their paper. It is also consistent with Fermi's having done it himself in 1950, when he again became interested in the problem. On the other hand, it is curious that Fermi would solve the radial equation numerically in 1947 and not go on to find the critical radius then. At any rate, all of this was unknown to those of us who became interested in the problem and recalculated $D_{\min }=0.639$ ea ea twenty years later. At least we know that the value has not changed in the intervening years!

\section{Acknowledgements}

I wish to thank the staff of the Special Collections Department of the University of Chicago Library, particularly Mary Janzen Wilson and Alice Eysenbach. Their courtesy, help, and patience made this article possible. Figure 11 and the photographs from Fermi's Notebooks were furnished by the Curator of Special Collections, University of Chicago Library. Teller's introduction to Papers 232 and 233 in Fermi's Collected Papers is reproduced here with the kind permission of the University of Chicago Press. I also want to thank H. C. Schweinler, W. R. Garrett, and Mary Janzen Wilson for reading this manuscript. A number of their comments and suggestions are included. I am very grateful to Mrs. Laura Fermi for permission to reproduce the material from Professor Fermi's Notebooks. 


\section{REFERENCES}

1. G. S. Hurst, J. A. Stockdale, and L. B. O'Kelly, J. Chem. Phys. 38, 2572 (1963).

2. Saul Altshuler, Phys. Rev. 107, 114 (1957).

3. J. E. Turner, Phys. Rev. 141, 21 (1966).

4. The atomic unit of the electric dipole moment is $e_{0}=2.54 \mathrm{x}$ $10^{-18}$ esu-cm, e being the electron's charge and $a_{0}$ the radius of the first Bohr orbit in hydrogen.

5. R. F. Wallis, R. Herman, and H. W. Milnes, J. Mnl. Spert. $\underline{4}, 51$ (1960).

6. 1 Rydberg $(k y)=e^{2} / 2 a_{0}=13.6 \mathrm{eV}$.

7. J. E. Turner and Kenneth Fox, "On the Bound States of an Electron in the Field of a Permanent Electric Dipole," ORNL-3895, Oak Ridge National Laboratory, Oak Ridge, TN 37830 (1965).

8. J. E. Turner and Kenneth Fox, J. Phys. Al, 118 (1968).

9. Kenneth Fox and J. E. Turner, Am. J. Phys. 34, 606 (1966).

10. Kenneth Fox and J. E. Turner, J. Chem. Phys. 45, 1142 (1966).

11. J. E. Turner, V. E. Anderson, and Kenneth Fox, Phys. Rev. 174, 81 (1968).

12. J. E. Turner and Kenneth rox, Phys. Letters 23,547 (1966).

13. W. Byers Brown and Robert E. Roberts, J. Chem. Phys. 46, 2006 (1967).

14. M. H. Mittleman and R. E. von Holdt, Phys. Rev. Al40, 726 (1965).

15. M. H. Mittleman and V. P. Myerscough, Phys. Letters 23, 545 (1966).

16. Jean Marc Lévy-Leblond, Phys. Rev. 153, 1 (1967).

17. 0. Crawford and A. Dalgarno, Chem. Phys. Letters 1, 23 (1967).

18. 0. H. Crawford, Proc. Phys. Soc. 91, 279 (1967). 
19. Cf. L. D. Landau and E. M. Lifshitz, Quantum Mechanics, 2nd ed., pp. 118-21 (Pergamon Press, 0xford, 1959).

20. C. A. Coulson and Mary Walmsley, Proc. Phys. Soc. 91, 31 (1967).

21. J. A. D. Stockdale, L. G. Christophorou, J. E. Turner, and

V. E. Anderson, Phys. Letters 25A, 510 (1967).

22. J. M. Levy-Leblond and J. P. Provost, Phys. Letters 26A, 104 (1967).

23. K. Takayanagi and Y. Itikawa, J. Phys. Soc. Japan 24, 160 (1968).

24. L. G. Christophorou and D. Pittman, J. Phys. B2, 71 (1969).

25. W. R. Garrett, Mo1. Phys. 24, 465 (1972).

26. A. S. Wightman, Phys. Rev. 77, 521 (1949).

27. E. Fermi and E. Teller, Phys. Rev. 72, 406 (1947).

28. The Collected Papers of Enrico Fermi, University of Chicago Press, Chicago (1965).

29. E. Teller and H. L. Sahlin, Physical Chemistry, An Advanced Treatise, Vol. V, Henry Eyring, ed., Academic Press, New York (1970).

30. The right-hand side of the equation near the bottom of Fig. 7 should read $\left(\varepsilon \rho^{2}-\varepsilon \mu^{2}+\beta \mu\right) \psi$.

31. The collection does contain some copies of correspondence between Fermi and the Physical Review, but I could find nothing related to this problem.

32. Initial contacts with Peter Adams of the Physical Review indicate that files on at least some old manuscripts may still exist in storage at Brookhaven. 


\section{FIGURE CAPTIONS}

Fig. 1. Summary of data (points) from Hurst, Stockdale, and 0'Kelly (Ref. 1) comapred with theory (solid line) of Altshuler (Ref. 2). The ordinate gives the ratio of thermal-energy electron scattering cross sections for various polar molecules in ethylene. The abscissa is in units of $(\text { esu-cm })^{2}$.

Fig. 2. Schematic representation of electron in stationary dipole field.

Fig. 3. Binding energy, $-\lambda$, as a function of dipole moment, $D$.

Fig. 4. First page of Fermi's work on dipole problem. Notebook 100 (D12), p. 285 .

Fig. 5. Separated equations. Notebook 100 (D12), p. 286.

Fig. 6. Condition on modified separation constant, $\gamma=-\alpha-\varepsilon$, for zero-energy solution. Notebook 100 (D12), p. 297.

Fig. 7. Sheet inserted on page 3 of Notebook 101 (D11), front side.

Fig. 8. Back side of inserted sheet. Notebook 101 (D11), p. 3.

Fig. 9. Notebook 101 (D11), p. 11

Fig. 10. Notebook 101 (DI1), p. 12.

Fig. 11. Reading room in the Special Collections Department, Joseph Regenstein Library, University of Chicago.

Fig. 12. The missing reference, "MG 3/904:"

Fig. 13. Index to Notehook 107 (D5).

Fig. 14. Fermi's calculation of the critical value, $\beta=1.28$. 
ORNL/TM-5440

INTERNAL DISTRIBUTION

$\begin{aligned} & \text { 1-2. } \text { Central Research Library } \\ & \text { 3. } \text { Document Reference Section } \\ & \text { 4-6. Laboratory Records } \\ & \text { 7. Laboratory Records, R.C. } \\ & \text { 8. ORNL Patent Office } \\ & \text { 9. } \text { E. T. Arakawa } \\ & \text { 10. } \text { J. C. Ashley } \\ & \text { 11. } \text { J. A. Auxier } \\ & \text { 12. L. G. Christophorou } \\ & \text { 13. R. N. Compton } \\ & \text { 14. W. R. Garrett } \\ & \text { 15. R. N. Hamm }\end{aligned}$

16. G. S. Hurst

17. M. G. Payne

18. C. R. Richmond

19. R. H. Ritchie

20. H. C. Schweinler

21. J. H. Thorngate

22-51. J. E. Turner

52. T. A. Welton

53. E. P. Wigner

54. $H:$ A. Wright

55. A. Zucker.

\section{EXTERNAL DISTRIBUTION}

56. Peter D. Adams, Physical Review, Brookhaven National Laboratory, Upton, NY 11973.

57. D. Arora, DRP, Bhabha Atomic Research Centre, Trombay, Bombay 400085, India.

58. J. S. Bisht, DRP, Bhabha Atomic Research Centre, Trombay, Bombay 400085 , India.

59. Werner Brandt,

60. W. Byers Brown, Theoretical Chemistry Institute, University of Wisconsin, Madison, WI 53706.

61. C. A. Coulson, Mathematical Institute, University of Oxford, Oxford, England.

62. 0. H. Crawford, Chemistry Department, Barnard College, Columbia University, New York, NY 10027.

63. A. Dalgarno, School of Physics and Applied Mathematics, The Queen's University of Belfast, Northern Ireland.

64. Alice Eysenbach, Joseph Regenstein Library, University of Chicago, Chicago, IL 60637.

65. U. Fano, Department of Physics, University of Chicago, Chicago, IL 60637.

66. Kenneth Fox, Department of Physics, University of Tennessee, Knoxville, TN 37916.

67. M. Inokuti, Argonne National Laboratory, Argonne, IL 60439.

68. Y. Itikawa, Institute of Space and Aeronautical Science, University of Tokyo, Komaba Meguro-ku, Tokyo, Japan.

69. R. K. Kerr, DRP, Bhabha Atomic Research Centre, Trombay, Bombay 400085 , India.

70. R. T. Lagemann, Department of Physics and Astronomy, Vanderbilt University, Nashville, TN 37215.

71. Jean Marc Lévy-Leblond, Laboraroire de Physique Théorique, Faculte des Sciences, 06-Nice, France.

72. M. H. Mittleman, Physics Department, City College, New York, NY 10031. 
73. K. Z. Morgan, School of Nuclear Engineering, Georgia Institute of Technology, Atlanta, GA 30332.

74. A. N. Nandakumar, DRP, Bhabha Atomic Research Centre, Trombay, Bombay 400085, India.

75. V. N. Neelavathi, DRP, Bhabha Atomic Research Centre, Trombay, Bombay 400085, India.

76. J. R. Peterson, Molecular Physics Center, Stanford Research Institute, Men1o Park, CA 94025.

77. Robert E. Roberts, Department of Chemistry, University of Indiana, Bloomington, IN 47401.

78. Theodore A. Roth, 17a Meadowbrook Village, Mays Landing, NJ 08330.

79. L. V. Spencer, Box 206, Gaithersburg, MD 20760.

80. K. Takayanagi, Institute of Space and Aeronautical Science, University of Tokyo, Komaba, Meguro-ku, Tokyo, Japan.

81. E. Teller, 1573 Hawthorneterr, Berkeley, CA 94708.

82. R. F. Wallis, Department of Physics, University of California, Irvine, CA 92664.

83. Spencer R. Weart, American Institute of Physics, 335 East 45 Street, New York, NY 10017.

84. Mary Janzen Wilson, Joseph Regenstein Library, University of Chicago, Chicago, IL 60637.

$8.5-$

111. Technical Information Center.

112. Research and Technical Support Division, ORO. 